\title{
Theoretical Investigation on New Analyzing Procedure of Anelastic Strain Recovery Method for Stress Measurements Based on Bayesian Statistical Modeling*1
}

\author{
Tatsuhiro Sugimoto*2, Kazuya Ishitsuka and Weiren Lin \\ Department of Urban Management, Graduate School of Engineering, Kyoto University, Kyoto 615-8540, Japan
}

\begin{abstract}
One significant issue associated with in situ stress measurements is that the uncertainty of the results cannot be determined. In this study, we propose a novel analytical procedure for the anelastic strain recovery (ASR) method, an in situ stress measurement method, enabling us to conduct uncertainty quantification based on Bayesian statistical modeling (BSM). The new procedure consists of the following steps: i) measuring the ASR of a rock core with strain gauges, ii) applying a probability model based on BSM to the measured ASR data and simulating the probability densities of the elements of an in situ stress tensor and other parameters; and iii) regarding the probability densities as the results of in situ stress measurements with uncertainty. This paper presents the results obtained by applying the proposed procedure to simulated ASR data. The results show that the uncertainties of some parameters are reduced by giving the elastic moduli. Notably, the rates of uncertainty decrease vary for each parameter. To reveal the cause of these differences, we introduce the new evaluation tool, Sobol' indices, which comprise a global sensitivity analytic tool, to facilitate a quantitative discussion. [doi:10.2320/matertrans.MT-Z2021015]
\end{abstract}

(Received August 6, 2021; Accepted September 2, 2021; Published October 15, 2021)

Keywords: anelastic strain recovery method, uncertainty quantification, Bayesian statistical modeling, in situ stress, rock core, global sensitivity analysis

\section{Introduction}

The in situ stress state in the earth's crust, which is a subject of rock mechanics, is crucial information in geosciences, such as structural geology and earth resource engineering. ${ }^{1,2)}$ A variety of methods have been proposed to measure crustal stress, such as the inversion of earthquake focal mechanisms, analysis of borehole breakout or drilling induced tensile fracture (DITF), hydraulic fracturing, and core-based methods. ${ }^{3)}$

One problem with stress measurements is that it is difficult to evaluate the uncertainty of the measurement results. Because it is generally difficult to perform stress measurements, multiple measurements at the same site and depth are not readily available. One possible solution is uncertainty quantification (UQ), which has been actively discussed in recent years within various fields of earth science. ${ }^{4)}$ Bayesian statistical modeling (BSM), a UQ method, enables us to evaluate uncertainty even with limited measurement data by utilizing information that is known before the measurement. The effectiveness of this method has been confirmed in the fields of earth and planetary sciences and rock mechanics for which there is generally a paucity of measurement data. $^{5-8)}$ However, no previous studies have investigated the application of UQ to stress measurements. In this study, we develop a new analytic procedure for the anelastic strain recovery (ASR) for UQ using BSM. The ASR method ${ }^{9)}$ is a core-based three-dimensional stress measurement method that can be applied to deep drilling (up to approximately $10 \mathrm{~km}$ depth) and has been used in recent onshore and offshore drilling projects. ${ }^{10-14)}$

Our overall research comprises two stages. In the first stage, we constructed a new ASR and evaluated the characteristics of the new analytic procedure using simulated

\footnotetext{
${ }^{* 1}$ This Paper was Originally Published in Japanese in J. Soc. Mater. Sci., Jpn. 70 (2021) 573-580.

${ }^{* 2}$ Graduate Student, Kyoto University
}

data. In the second stage, we will apply the method to appropriate real data and verify its validity. We report on the first stage of the study herein, expanding the theory of BSM applied to ASR stress measurement and presenting the results obtained by the new procedure as applied to simulated ASR data.

\section{ASR Method}

This chapter describes the constitutive equation of ASR and the analytic procedure currently in use (hereinafter referred to as the "conventional method").

A rock core that is released from in situ stress at the time of drilling is generally subjected to an instantaneous elastic strain recovery followed by a time-dependent strain recovery that takes several days to several weeks to complete, as shown in Fig. 1(a). The latter is called the ASR. ASR is usually measured by applying strain gauges to the rock core immediately after drilling.

A three-dimensional in situ stress tensor is calculated based on the constitutive equation relating the time-dependent strain recovery in multiple directions to the in situ stress tensor

$$
\varepsilon(t, \mathbf{n})=\left(\sigma_{i j} n_{i} n_{j}-\sigma_{\mathrm{m}}\right) J_{\mathrm{S}}(t)+\left(\sigma_{\mathrm{m}}-p_{0}\right) J_{\mathrm{V}}(t),
$$

which is derived by Matsuki and Takeuchi. ${ }^{9)}$ In this paper, unless otherwise stated, the subscripts of vectors and tensors take values from 1 to 3 , and if the same subscript appears in the same term, the sum of 1 to 3 is taken. In eq. (1), $t$ is the time elapsed from the start of the ASR measurement, $\mathbf{n}=\left(n_{1}, n_{2}, n_{3}\right)^{\mathrm{T}}$ is the directional cosine vector of the direction of measurement of anelastic normal strain recovery (nine directions shown in Fig. 1(b)), $\varepsilon$ is the anelastic normal strain recovery in the $\mathbf{n}$ direction, $\sigma_{i j}$ is the three-dimensional in situ stress tensor, $\sigma_{\mathrm{m}}=\sigma_{l l} / 3$ is the mean normal stress, and $p_{0}$ is the pore pressure. $J_{\mathrm{S}}$ and $J_{\mathrm{V}}$ are the ASR compliances of shear and volumetric deformation, respectively, which are defined by the following equations: 
(a)

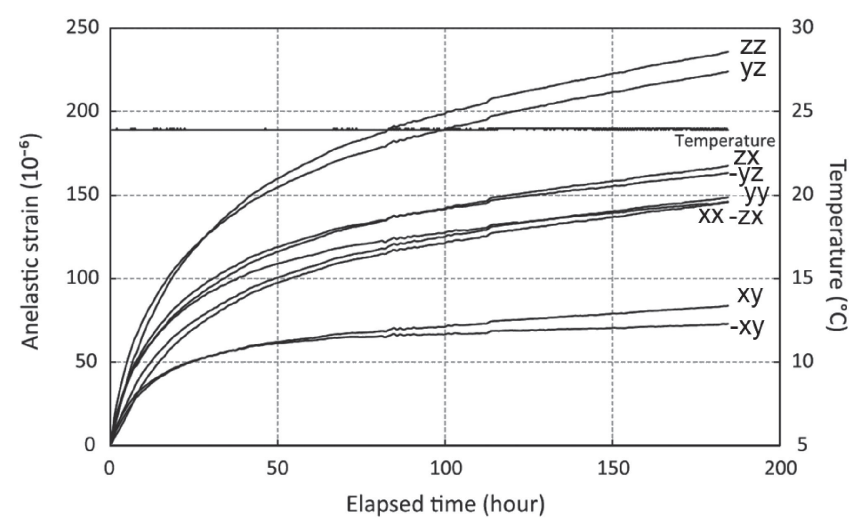

(b)

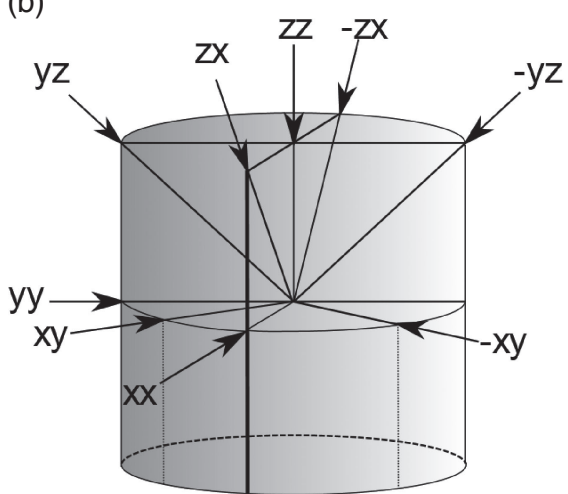

Fig. 1 (a) Real anelastic normal strain recovery data measured on a sedimentary rock core sample collected in the eastern Nankai Trough (modified from Nagano et al. ${ }^{13)}$ ). The labels ( $x x, x y$ and so on) indicate the nine directions in which anelastic normal strains were measured and correspond to those shown in (b). The temperature of measured samples is also shown in the figure. (b) Nine directions in which anelastic normal strains are measured with strain gauges. The directions of $\pm x y, \pm y z, \pm z x$ are tilted at 45 degrees with respect to those of $x x, y y, z z$.

$$
\begin{aligned}
& J_{\mathrm{S}}(t)=\frac{e_{i j}(t)}{s_{i j}} \\
& J_{\mathrm{V}}(t)=\frac{\varepsilon_{\mathrm{m}}(t)}{\left(\sigma_{\mathrm{m}}-p_{0}\right)},
\end{aligned}
$$

where $e_{i j}=\varepsilon_{i j}-\varepsilon_{\mathrm{m}} \delta_{i j}$ is the anelastic deviatoric strain tensor, $\varepsilon_{i j}$ is the ASR tensor, $\varepsilon_{\mathrm{m}}=\varepsilon_{l l} / 3$ is the anelastic mean normal strain recovery, and $s_{i j}=\sigma_{i j}-\sigma_{\mathrm{m}} \delta_{i j}$ is the deviatoric stress tensor. In eq. (1) and eq. (2), it is assumed that the in situ stress and pore pressure are released immediately after drilling. When the rock core can be regarded as an isotropic linear viscoelastic material, it is shown that the directions of the principal strains of ASR coincide with those of the principal stresses. The three directions of the principal strains can be determined using the least-squares method for anelastic normal strain recovery in six independent directions measured with strain gauges. In the conventional method, these three principal strain directions are used as the directions of the principal stresses.

The magnitudes of three principal stresses are obtained from

$$
\sigma_{i}=\frac{e_{i}(t)}{J_{\mathrm{S}}(t)}+\frac{\varepsilon_{\mathrm{m}}(t)}{J_{\mathrm{V}}(t)}+p_{0},
$$

where $\sigma_{i}$ and $e_{i}(i=1,2,3)$ represent the principal stress and anelastic principal deviatoric strain recovery, respectively. Here, stress is defined as $\sigma_{1}>\sigma_{2}>\sigma_{3}$ with compression being positive, and strain recovery is defined as $e_{1}>e_{2}>e_{3}$ with expansion being positive. In the conventional method, the stress tensor can be calculated by determining $J_{\mathrm{S}}, J_{\mathrm{V}}$, and $p_{0}$ in addition to $\varepsilon_{i j}$. ASR compliances $J_{\mathrm{S}}$ and $J_{\mathrm{V}}$ should be measured by conducting a triaxial compression test on the rock core used for the ASR measurement. However, it has been suggested by Matsuki and Takeuchi ${ }^{9)}$ that ASR compliances depend on the magnitude of mean normal stress, which generally makes the measurement difficult to conduct in the laboratory. In the conventional method, therefore, the in situ stress tensor is calculated using the ratio of ASR compliances $r=J_{\mathrm{V}} / J_{\mathrm{S}}$, vertical stress $\sigma_{\mathrm{v}}$ and pore pressure $p_{0}$. These values are determined as follows: Using eq. (3), the vertical stress $\sigma_{\mathrm{v}}$ is calculated as follows:

$$
\begin{aligned}
\sigma_{\mathrm{v}} & =\sigma_{1} l_{1}^{2}+\sigma_{2} l_{2}^{2}+\sigma_{3} l_{3}^{2} \\
& =\frac{1}{J_{\mathrm{V}}(t)}\left(r(t) l_{i} l_{j} e_{i j}(t)+\varepsilon_{\mathrm{m}}(t)\right)+p_{0} .
\end{aligned}
$$

In eq. (4), the principal strain coordinate system is used as the basis, and $\mathbf{I}=\left(l_{1}, l_{2}, l_{3}\right)^{\mathrm{T}}$ is the directional cosine vector in the vertical direction with respect to the coordinate system. ASR compliances can be expressed using $r$ and $\sigma_{\mathrm{v}}$

$$
\begin{aligned}
J_{\mathrm{V}}(t) & =\frac{\left(r(t) l_{i} l_{j} e_{i j}(t)+\varepsilon_{\mathrm{m}}(t)\right)}{\left(\sigma_{\mathrm{V}}-p_{0}\right)} \\
J_{\mathrm{S}}(t) & =\frac{1}{r(t)} J_{\mathrm{V}}(t) .
\end{aligned}
$$

In the conventional method, $r$ is usually assumed to be the constant $r_{\mathrm{c}}$, and $r_{\mathrm{c}}=0.64 \pm 0.14$, which is the value at $14 \mathrm{~h}$ after measurement was initiated, as determined by Matsuki ${ }^{15)}$ for six rocks, is used. In eq. (5), it is also necessary to determine the values of $\sigma_{\mathrm{v}}$ and $p_{0}$. In a previous study, ${ }^{12)}$ the vertical stress $\sigma_{\mathrm{v}}$ was assumed to be equal to $\rho_{\mathrm{r}} g h$, the overburden stress was calculated using the average wet density of the rocks above the measurement point (sampling depth), and the pore pressure $p_{0}$ was assumed to be equal to the hydrostatic pressure $\rho_{\mathrm{w}} g h_{\mathrm{w}}$, where $g$ is the gravitational acceleration, $h$ is the depth of the measurement point, $h_{\mathrm{w}}$ is the distance between the measurement point and the groundwater surface, and $\rho_{\mathrm{r}}$ and $\rho_{\mathrm{W}}$ are the average wet density of the rock and water density, respectively.

\section{Bayesian Statistical Modeling}

In this chapter, we review the general theory of BSM. The new ASR analytic procedure is described in detail in the next chapter. $\mathrm{BSM}^{16)}$ is a parameter estimation method that represents the estimation results by a posterior distribution

$$
\Pi(\mathbf{w} \mid D)=\frac{L(D \mid \mathbf{w}) \phi(\mathbf{w})}{Z(D)}
$$


where $\mathbf{w}=\left(w_{1}, w_{2}, \cdots, w_{q}\right)^{\mathrm{T}}$ is a vector of $q$ parameters to be estimated, and $D$ is the observed data. $Z$ is a normalizing constant defined by:

$$
Z(D)=\int_{\Omega} \mathrm{d} \tau L(D \mid \tau) \phi(\tau) .
$$

$\Omega \subset \mathbb{R}^{q}$ is the set of all possible values of parameters ( $\mathbb{R}$ is the set of real numbers), $L$ is called a likelihood function, which shows the probability of obtaining data $D$ given a set of values of parameters. It takes various forms depending on the problem to be applied. The likelihood function used in this study is explained in the next chapter. $\phi$ is a probability density function called the prior distribution, which needs to be determined for each parameter. Although there have been various discussions on how to determine the prior distribution, ${ }^{17)}$ in this study, we set the distribution based on the information obtained in the actual application. The details are described in Section 4.3.

Because the posterior distribution $\Pi$ is a joint probability distribution for all parameters, it is necessary to calculate the marginal posterior distribution (MPD)

$$
\pi_{i}\left(w_{i} \mid D\right)=\int \mathrm{d} w_{1} \cdots \mathrm{d} w_{i-1} \mathrm{~d} w_{i+1} \cdots \mathrm{d} w_{q} \Pi(\mathbf{w} \mid D)
$$

to obtain the results for each parameter. However, it is generally difficult to obtain posterior distribution and MPDs analytically; therefore, they are often simulated by Markov Chain Monte Carlo (MCMC). ${ }^{18)}$ There are many excellent texts on MCMC, ${ }^{19,20)}$ so we do not describe the details and provide only a brief overview.

In MCMC, a Markov process with a posterior distribution as the stationary distribution is generated on a computer, and the MPDs are obtained by sampling for each parameter from the Markov process. The most important point is whether the obtained sample sequences of each parameter converge to the true MPDs. There have been various methods for assessing convergence, and in this study, we adopted the widely used method of Gelman et al. ${ }^{21)}$ In this method, the convergence decision is made according to the values

$$
\hat{R}=\sqrt{\frac{N-1}{N}+\frac{1}{N} \frac{B}{W}}
$$

computed for each parameter from the multiple sample sequences with different initial values, where $N$ is the number of steps in the sample sequence (number of samples included), $B$ is the variance between sample sequences, and $W$ is the variance within a sample sequence (for the exact definition, see Gelman et al. ${ }^{22)}$ ). In this method of determining convergence, we conclude that convergence has been achieved when the variation between multiple sample sequences starting from different initial values is less than the variation within each sample sequence. Gelman et al. ${ }^{22)}$ proposed a criterion in which convergence is judged when there are three or more sample sequences and $\hat{R}<1.1$ for all parameters, and the same criterion was used in this study. In this study, the MCMC was executed using the open source software Stan. ${ }^{23)}$

The advantages of BSM are that it provides a flexible approach for using information that is known prior to the measurement in the form of prior distributions and quantitatively determines uncertainty as a probability distribution.

\section{New ASR Method Analytic Procedure}

In this chapter, we present a new analytic procedure of the ASR method based on BSM (hereinafter referred to as the "new method"). In the new method, the in situ stress is estimated by applying a probabilistic model, which consists of a likelihood function and a prior distribution, to the anelastic normal strain recovery data. The results of the estimation are used as the results of the in situ stress measurement. In the following sections, we provide detailed explanations on data, likelihood functions, and prior distributions used.

\subsection{Data}

The data used in the new method are the same as those used in the conventional method (Fig. 1(a)), which is the time series data $D=\left\{\left(t_{i}, \boldsymbol{\varepsilon}_{i}\right) \mid i=1 \sim m\right\}$ of anelastic normal strain recovery measured with strain gauges. $t_{i}$ is the time elapsed from the start of the measurement, $\boldsymbol{\varepsilon}_{i}=$ $\left(\varepsilon_{i}^{1}, \varepsilon_{i}^{2}, \cdots, \varepsilon_{i}^{d}\right)^{\mathrm{T}}$ is a vector of the anelastic normal strain recovery data for $d$ directions (up to nine directions shown in Fig. 1(b)), and $m$ is the number of sampling points of the anelastic normal strain recovery data at time $t_{i}$. For details of the measurement procedure and system, see Lin et al. ${ }^{10)}$

In this study, we used simulated data rather than real data to examine the new method. The details of the simulated data are shown in Chapter 5.

\subsection{Likelihood function}

The likelihood function in this study consists of two components: (i) eq. (1), which is a constitutive equation relating the anelastic normal strain recovery to the in situ stress tensor, and (ii) the probability density function to which the measurement error of anelastic normal strain recovery is subjected. In the conventional method, stress is calculated without assuming specific functional forms of ASR compliances in eq. (1), but the new method uses specific functional forms of these to calculate the in situ stress. In this study, we use

$$
\begin{aligned}
& J_{\mathrm{S}}(t)=\frac{1}{2 G}\left(1-\exp \left(-\frac{t}{\tau_{\mathrm{s}}}\right)\right) \\
& J_{\mathrm{V}}(t)=\frac{1}{3 K}\left(1-\exp \left(-\frac{t}{\tau_{\mathrm{V}}}\right)\right),
\end{aligned}
$$

which are obtained by assuming a three-element model consisting of two springs and one dashbod. ${ }^{24)} G$ and $K$ are the shear modulus and bulk modulus of the rock comprising the measured rock core, $\tau_{\mathrm{s}}$ and $\tau_{\mathrm{v}}$ are the retardation times for the shear and volumetric deformation of the rocks, defined by

$$
\begin{gathered}
\tau_{\mathrm{S}}=\frac{\eta_{\mathrm{S}}}{2 G} \\
\tau_{\mathrm{V}}=\frac{\eta_{\mathrm{V}}}{3 K},
\end{gathered}
$$

where $\eta_{\mathrm{S}}$ and $\eta_{\mathrm{V}}$ represent the shear and volumetric viscosities of the rock, respectively. The retardation time is the time required for strain recovery to reach $63.2 \%$ of the 
Table 1 Parameters, their values used for making simulated data, and prior distributions for each case.

\begin{tabular}{|c|c|c|c|c|}
\hline \multirow[t]{2}{*}{ Parameter } & \multirow[t]{2}{*}{ Symbol } & \multirow[t]{2}{*}{ Value } & \multicolumn{2}{|c|}{ Prior distribution } \\
\hline & & & Case-1 & Case-2 \\
\hline \multirow{6}{*}{ Stress tensor $[\mathrm{MPa}]$} & $\sigma_{11}$ & 35 & $\mathrm{U}[0,100]$ & $\mathrm{U}[0,100]$ \\
\hline & $\sigma_{22}$ & 30 & $\mathrm{U}[0,100]$ & $\mathrm{U}[0,100]$ \\
\hline & $\sigma_{33}$ & 37 & $\mathrm{~N}[37,5]$ & $\mathrm{N}[37,5]$ \\
\hline & $\sigma_{12}$ & 1 & $\mathrm{U}[0,100]$ & $\mathrm{U}[0,100]$ \\
\hline & $\sigma_{13}$ & 2 & $\mathrm{U}[0,100]$ & $\mathrm{U}[0,100]$ \\
\hline & $\sigma_{23}$ & 3 & $\mathrm{U}[0,100]$ & $\mathrm{U}[0,100]$ \\
\hline Pore pressure $[\mathrm{MPa}]$ & $p_{0}$ & 15 & $\mathrm{~N}[15,5]$ & $\mathrm{N}[15,5]$ \\
\hline Shear modulus [GPa] & $G$ & 30 & $\mathrm{U}[0, K]$ & 30 \\
\hline Bulk modulus [GPa] & $K$ & 50 & $\mathrm{U}[0,100]$ & 50 \\
\hline \multirow{2}{*}{ Retardation time [hour] } & $\tau_{\mathrm{V}}$ & 13.33 & $\mathrm{U}[0,100]$ & $\mathrm{U}[0,100]$ \\
\hline & $\tau_{\mathrm{S}}$ & 22.68 & $\mathrm{U}[0,100]$ & $\mathrm{U}[0,100]$ \\
\hline Variance $\left[10^{-6}\right]$ & $s$ & & $\mathrm{U}[0,100]$ & $\mathrm{U}[0,100]$ \\
\hline
\end{tabular}

total ASR. The ASR compliances used in this study have not yet been formulated to simulate dependence on the mean normal stress; therefore, the modification of ASR compliances (10) requires further study. The probability density function that the measurement error of the anelastic normal strain recovery obeys is considered to be a normal distribution. These conditions lead to a likelihood function

$$
\begin{aligned}
L(D \mid \mathbf{w})= & \prod_{i=1}^{m} \frac{1}{\sqrt{(2 \pi)^{d}|\boldsymbol{\Sigma}|}} \\
& \times \exp \left[-\frac{1}{2}\left(\boldsymbol{\varepsilon}_{i}-\overline{\boldsymbol{\varepsilon}}\left(t_{i}\right)\right)^{\mathrm{T}} \boldsymbol{\Sigma}^{-1}\left(\boldsymbol{\varepsilon}_{i}-\overline{\boldsymbol{\varepsilon}}\left(t_{i}\right)\right)\right],
\end{aligned}
$$

where $\boldsymbol{\varepsilon}_{i}$ is a vector with the measured data as elements, $\overline{\boldsymbol{\varepsilon}}$ is a vector with the theoretical value of the anelastic normal strain recovery calculated from eq. (1), and $\Sigma$ is a covariance matrix of multivariate normal distribution to which the measurement error of the anelastic normal strain recovery obeys. In this study, the measurement error of the anelastic normal strain recovery was assumed to be constant regardless of the direction of measurement, and the measurement in each direction was independent. Therefore, $\boldsymbol{\Sigma}=s \mathbf{I}$ was used, where $s$ is a scalar representing the value of the variance that is common in all measurement directions, and $\mathbf{I}$ is the $d$ dimensional unit matrix. The parameter to be estimated is $\mathbf{w}=\left(\sigma_{11}, \sigma_{22}, \sigma_{33}, \sigma_{12}, \sigma_{13}, \sigma_{23}, p_{0}, G, K, \tau_{\mathrm{S}}, \tau_{\mathrm{V}}, s\right)^{\mathrm{T}}$.

\subsection{Prior distribution}

The method for determining the prior distribution has been debated, as mentioned above; in this study, we propose that the prior distributions are determined based on the information obtained separately from the stress measurement (prior information). In the following, we explain three sets of prior information and prior distributions derived from prior information.

\subsubsection{Prior information 1}

The ranges of realistic values of each parameter were used as a priori information. The ranges of stress and pore pressure in the depth interval where the ASR method can be applied is from $0 \mathrm{MPa}$ to $100 \mathrm{MPa}$. The possible ranges of the elastic moduli $G$ and $K$ are from $0 \mathrm{GPa}$ to $100 \mathrm{GPa}$, and the possible ranges of retardation times $\tau_{\mathrm{S}}$ and $\tau_{\mathrm{V}}$ are from $0 \mathrm{~h}$ to $100 \mathrm{~h}$. $s$, which represents the measurement error, is $>0$. From the theory of elasticity, we can conclude that $K>G$. Based on this prior information, we define prior distributions for each parameter. These are summarized in Table 1.

\subsubsection{Prior information 2}

The vertical element of the stress tensor at the measurement depth is close to the overburden stress calculated from the average wet density of rocks that exist above the measurement depth, and the pore pressure at the same depth is expected to be close to the hydrostatic pressure calculated from the groundwater level. This prior information can be expressed as probability density functions of the vertical element of the stress tensor, and the pore pressure with the mean is the overburden pressure and hydrostatic pressure, respectively. In this study, the aforementioned prior information is expressed by setting the prior distribution of $\sigma_{33}$, which is the vertical element in the coordinate system used in this study, as a normal distribution with a mean of $\rho_{\mathrm{r}} g h$ and a standard deviation of $5 \mathrm{MPa}$, and by setting the prior distribution of $p_{0}$ as the normal distribution with the mean $\rho_{\mathrm{w}} g h_{\mathrm{w}}$ and standard deviation of $5 \mathrm{MPa}$. Note that the values of $\rho_{\mathrm{r}} g h$ and $\rho_{\mathrm{w}} g h_{\mathrm{w}}$ are the true values of $\sigma_{33}$ and $p_{0}$ (Value in Table 1), which are described in the next chapter.

\subsubsection{Prior information 3}

In some cases, measurements of the elastic moduli $G$ and $K$ can be obtained from experiments. We consider using these measurements as prior information. In this study, we represent this prior information by using $G$ and $K$ as constants rather than parameters to be estimated. In this study, the true values of $G$ and $K$ in Table 1 , which are described in the next chapter, are used as measurements of the moduli.

The information available varies from case to case, and many combinations of information are available for actual applications of stress measurement. Therefore, this paper focuses on the following two patterns of case studies 


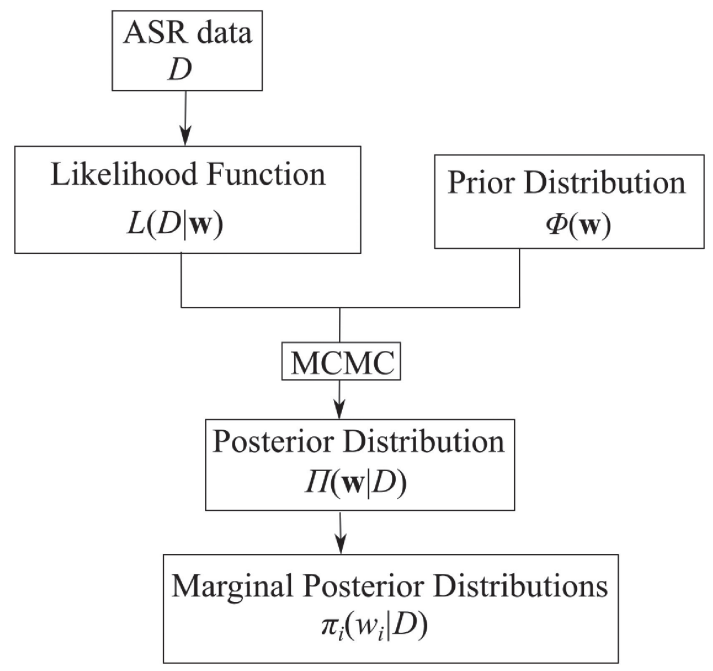

Fig. 2 Flow chart showing the concept of new analyzing procedure proposed in this study.

(combinations of prior information) to estimate the stress values. The first case is that in which both prior information 1 and 2 are available, which we call Case-1. The second case is that in which all prior information $(1,2$, and 3$)$ is available, which we call Case-2. The prior distributions of each parameter are shown in Table 1 for Case-1 and Case-2, respectively, where $\mathrm{U}[a, b]$ denotes a uniform distribution from $a$ to $b$, and $\mathrm{N}[c, d]$ denotes a normal distribution with mean $c$ and standard deviation $d$, respectively.

\subsection{Summary of the new method}

The flow of the stress measurement in the new method is summarized as follows: (i) the anelastic normal strain recovery of a rock core is measured in the same manner as in the conventional method, (ii) eq. (12) and the prior distributions shown in Table 1 are applied to the measured anelastic normal strain recovery data, and then sampling by MCMC to obtain the MPDs of each parameter, and (iii) the obtained MPDs are regarded as the measurement results of in situ stress and other parameters. Figure 2 shows the flow.

\section{Simulated ASR Data}

In the first stage of this study, the new method was examined using simulated data of ASR generated from eq. (1). The values of the parameters used to create the simulated data are listed in Table 1 . The principal stress values in this setting are $\sigma_{1}=39 \mathrm{MPa}$ (the maximum principal stress), $\sigma_{2}=34 \mathrm{MPa}$ (the intermediate principal stress), and $\sigma_{3}=29 \mathrm{MPa}$ (the minimum principal stress), respectively, and the direction of the intermediate principal stress is set to be subparallel to the vertical direction. The stresses and pore pressures were determined on the basis of the values around $1.5 \mathrm{~km}$ depth, and the coefficients of viscoelasticity of the rocks were determined on the basis of several references, ${ }^{15,25,26)}$ assuming that the rocks are granite. The true values of the parameters estimated in this study are shown in Table 1. To the simulated data, a random number following a normal distribution (mean 0 , standard deviation

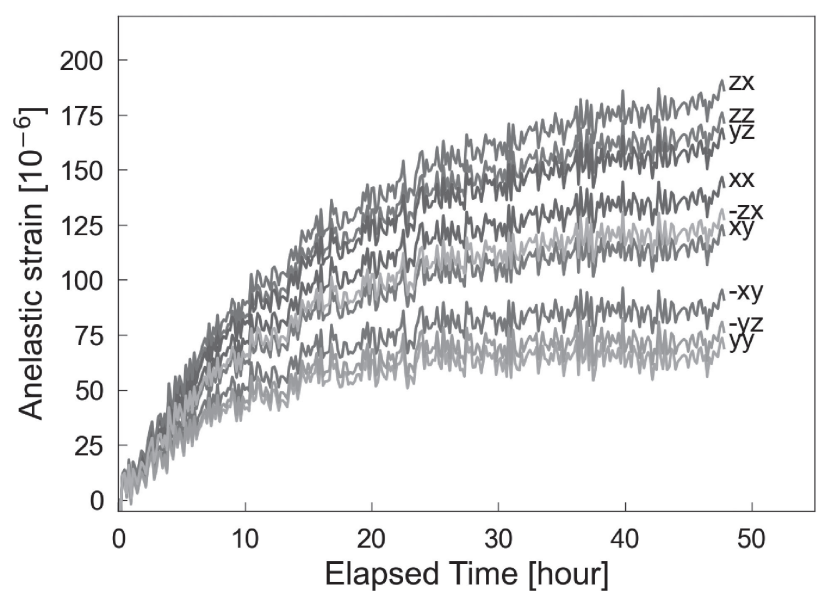

Fig. 3 Simulated ASR data developed by using eq. (1) and values shown in Table 1. The labels ( $x x, x y$, and so on) correspond to those shown in Fig. 1(b).

$5 \times 10^{-6}$ ) was added as a measurement error. The simulated data using the values in Table 1 are shown in Fig. 3. We used $48 \mathrm{~h}$ of data from the start of the measurement for estimation to limit computational costs. The sampling interval was set to $10 \mathrm{~min}$, which is the same as the actual measurement, and the number of sampling points was 288 in each direction.

\section{Results}

The MCMC in this study was performed using a supercomputer owned by Kyoto University, and a single simulation took approximately two hours. Figure 4 shows the estimation results of $\sigma_{11}$ in Case-1. Figure 4(a) shows the three sample sequences obtained by MCMC, and Fig. 4(b) shows the resulting MPD. Each sample sequence consisted of 1500 samples, giving a total of 4500 samples for each parameter. The three sample sequences are programmed to start from different initial values, and in Fig. 4(a), the sampling depends on the initial values up to approximately 100 steps. The initial value is the sample selected using a random number (independent of the probability model in

(a)

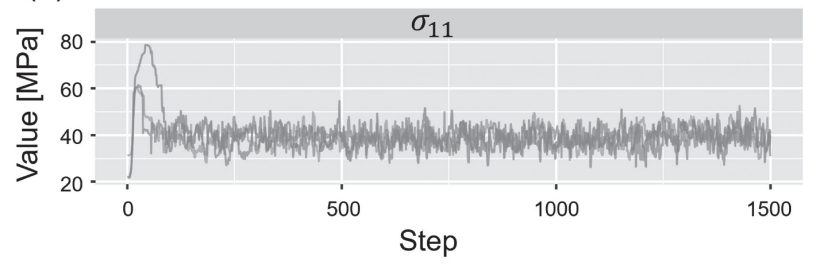

(b)

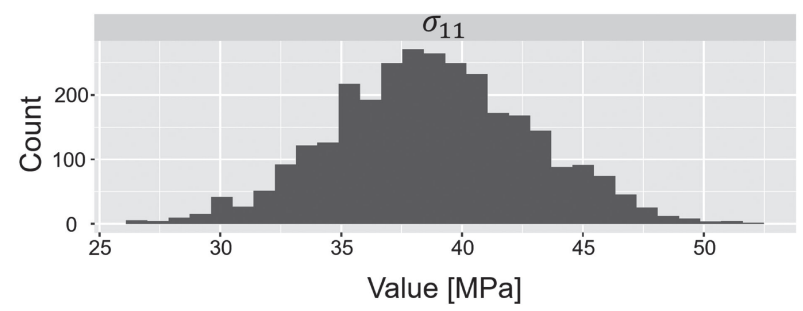

Fig. 4 Results of inference for $\sigma_{11}$ in Case-1. (a) Three Markov chains with different initial values. Each chain consists of 1500 samples. (b) Marginal posterior distribution obtained from three chains in (a). 
Table 2 Results of inference. True values are the same as those shown in Table 1. Mean and SD are the mean values and standard deviations calculated using the three chains excluding the warm-up samples.

\begin{tabular}{|c|c|c|c|c|c|c|c|c|c|c|}
\hline \multirow[t]{2}{*}{ Symbol } & \multirow[t]{2}{*}{ Unit } & \multirow[t]{2}{*}{ True value } & \multicolumn{4}{|c|}{ Case-1 } & \multicolumn{4}{|c|}{ Case-2 } \\
\hline & & & Mean & SD & Deviance & Rhat & Mean & SD & Deviance & Rhat \\
\hline$\sigma_{11}$ & \multirow{6}{*}{ [MPa] } & 35 & 38.77 & 4.07 & 3.77 & 1.01 & 35.07 & 3.42 & 0.07 & 1.00 \\
\hline$\sigma_{22}$ & & 30 & 28.60 & 3.93 & 1.40 & 1.02 & 30.07 & 3.42 & 0.07 & 1.00 \\
\hline \multirow{2}{*}{$\begin{array}{l}\sigma_{33} \\
\sigma_{12}\end{array}$} & & 37 & 42.84 & 4.37 & 5.84 & 1.01 & 37.07 & 3.42 & 0.07 & 1.00 \\
\hline & & 1 & 2.03 & 0.39 & 1.03 & 1.02 & 1.00 & 0.02 & 0.00 & 1.00 \\
\hline \multirow{2}{*}{$\begin{array}{l}\sigma_{13} \\
\sigma_{23}\end{array}$} & & 2 & 4.07 & 0.78 & 2.07 & 1.02 & 2.00 & 0.03 & 0.00 & 1.00 \\
\hline & & 3 & 6.10 & 1.17 & 3.10 & 1.02 & 3.00 & 0.04 & 0.00 & 1.00 \\
\hline$p_{0}$ & {$[\mathrm{MPa}]$} & 15 & 9.28 & 3.88 & 5.72 & 1.02 & 15.14 & 3.42 & 0.14 & 1.00 \\
\hline \multirow{2}{*}{$\begin{array}{l}G \\
K \\
\end{array}$} & \multirow{2}{*}{ [GPa] } & 30 & 60.95 & 11.71 & 30.95 & 1.02 & & & & \\
\hline & & 50 & 72.50 & 10.50 & 22.50 & 1.01 & & & & \\
\hline \multirow{2}{*}{$\begin{array}{l}\tau_{\mathrm{V}} \\
\tau_{\mathrm{S}}\end{array}$} & \multirow{2}{*}{ [hour] } & 13.33 & 13.23 & 0.07 & 0.10 & 1.00 & 13.23 & 0.08 & 0.10 & 1.00 \\
\hline & & 22.68 & 22.74 & 0.54 & 0.06 & 1.00 & 22.74 & 0.53 & 0.06 & 1.00 \\
\hline$s$ & {$\left[10^{-6}\right]$} & & 22.53 & 0.62 & & 1.00 & 22.48 & 0.60 & & 1.00 \\
\hline
\end{tabular}

use), and the number of steps is counted with the initial value as the first. In general, samples up to several hundred steps are not used in the analysis because they are dependent on the initial values. This part of the sample is called the warm-up. In this study, we decided to define the warm-up as the samples up to 500 steps and not to use them for further analysis. Therefore, the following analysis was performed with 3000 samples for each parameter. The estimation results for Case- 1 and Case-2 are shown in Table 2. The table lists the mean (Mean), standard deviation (SD), and $\hat{R}$ values calculated using 3000 samples, excluding the warm-up of each parameter.

In this study, we discuss the estimation results from two points of view: (i) the uncertainty of the estimation results and (ii) the difference between the estimated value and the true value. The standard deviation of the MPD (SD in Table 2) is used as the measure of (i), and the difference between the mean and true values (the difference between the true value and Mean in Table 2) is used as the measure of (ii). In this paper, we call the former uncertainty and the latter deviance, and we evaluate the estimation in which both uncertainty and deviance are small as good estimations.

In Case-1, the elastic moduli are assumed to be unmeasured and are parameters for estimation. The estimation results of the stress tensor (Table 2) show that the uncertainty of the non-diagonal elements $\left(\sigma_{12}, \sigma_{13}\right.$, and $\left.\sigma_{23}\right)$ are smaller than those of the diagonal elements $\left(\sigma_{11}, \sigma_{22}\right.$, and $\left.\sigma_{33}\right)$, while there is no significant difference in deviance between the diagonal and non-diagonal elements. The results of the estimates of the shear and bulk moduli show that both uncertainty and deviance are greater than the other parameters. Conversely, the retardation times, which represent the viscoelastic properties, are estimated to be better than other parameters.

We next examine the results of Case-2, where the elastic moduli are no longer parameters for estimation because it is assumed to be given in Case-2. The results of the stress tensor estimation show that the uncertainty of the non-diagonal elements is significantly less than that of the diagonal elements. Furthermore, unlike Case-1, the non-diagonal elements of the deviance are smaller, indicating that we can estimate the true value more accurately.

Comparing the two cases, we can conclude that the deviances are smaller in Case-2 than in Case-1 for all parameters. In other words, the probability model using the constitutive equation given by eq. (10) provides a more accurate estimate of the true value by providing the elastic moduli as prior information. This is an intuitively valid result. In contrast, uncertainty in the non-diagonal element of the stress tensor decreases from Case- 1 to Case-2, yet the decrease is hardly observed in the diagonal element and the pore pressure compared to the non-diagonal element. In the estimation results of retardation times, there is almost no difference between Case-1 and Case-2, and both are considered to exhibit good estimation.

These results indicate that the quality of the estimation differs from one parameter to another. A possible reason for the differences between parameters is the difference in the appearance of the parameters in eq. (1), which is the basis of the estimation. In other words, the differences in the importance of each parameter in eq. (1) are supposed to result in different estimation results. Sensitivity analysis provides a framework to quantitatively evaluate the importance of parameters. In the next chapter, the differences in each parameter are discussed based on the sensitivity analysis.

\section{Discussion Based on Sensitivity Analysis}

First, an overview of the sensitivity analysis is briefly described. A detailed explanation is presented elsewhere (e.g., Ref. 27)). Given the function $y=f(\mathbf{x})$, where $\mathbf{x}=$ $\left(x_{1}, x_{2} \cdots, x_{q}\right)^{\mathrm{T}}$ is a parameter of this model. Sensitivity analysis provides a framework for quantitatively assessing the contribution of $\mathbf{x}$ to the variation in $y$ caused by variation in the parameter $\mathbf{x}$. It has been pointed out that when performing parameter estimation, parameters with higher sensitivity can be estimated better than those with lower sensitivity. ${ }^{28)}$ In this paper, we present an analysis using the Sobol' method, ${ }^{29)}$ a widely used sensitivity analysis in which 
(a) $x x$ direction

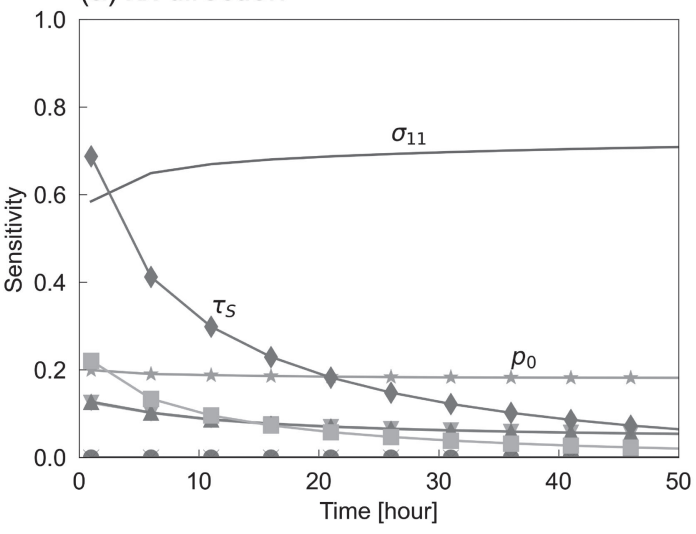

(b) xy direction

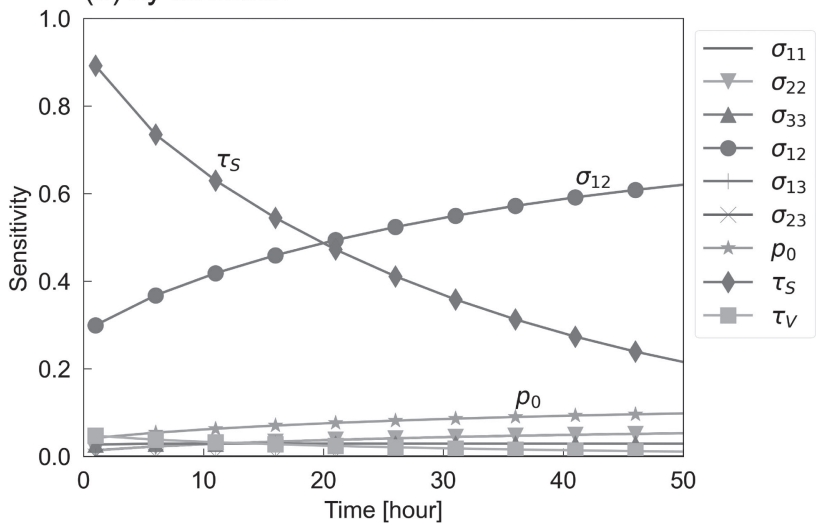

Fig. 5 Total sensitivity indices for each parameter in Case-2. (a) Sensitivities calculated using anelastic normal strain recovery in the $x x$ direction in Fig. 1(b). (b) Sensitivities calculated using anelastic normal strain recovery in the xy direction in Fig. 1(b).

the contribution of the variance of $x_{i}$ to the variance of $y$ is used as a sensitivity index. In this study, we use the Total Sensitivity Indices $S_{\mathrm{T}},{ }^{30}$ ) which are used in Sobol' method and consider the interaction of all parameters. In particular, we conducted a sensitivity analysis of Case-2, which shows a significant difference in uncertainty. Figure 5 shows the $S_{\mathrm{T}}$ of each parameter calculated using eq. (1). Figure 5(a) and (b) show the results when $\mathbf{n}=(1,0,0)^{\mathrm{T}}$ is substituted in eq. (1) (direction $x x$ ), and $\mathbf{n}=\left(\cos 45^{\circ}, \sin 45^{\circ}, 0\right)^{\mathrm{T}}$ is substituted in eq. (1) (direction $x y$ ), respectively. The horizontal axis is the time elapsed since the start of measurement, and the figures show the value of sensitivity at each time.

For each element of the stress tensor and the pore pressure, the sensitivity of the diagonal element (in this case, $\sigma_{11}$ ) is large in absolute value in the $x x$ direction, but the sensitivity of the pore pressure is relatively large compared to that of $\sigma_{11}$. Conversely, for the data in the $x y$ direction, the sensitivity of the non-diagonal element (in this case, $\sigma_{12}$ ) is relatively greater than the sensitivity of the other parameters. In this $x x$ direction, the sensitivity of parameters other than the diagonal element is also relatively large, which indicates that the uncertainty is greater than that of the non-diagonal element. Because the retardation time represents the time constant of the strain recovery curve, it can be expected that the sensitivity increases in the early part of the measurement, where the curvature is large. In fact, the sensitivity of $\tau_{\mathrm{S}}$ increases at the beginning of the measurement. However, it can be observed that the sensitivity of $\tau_{\mathrm{V}}$ is zero most of the time. This suggests that low sensitivity is not necessarily associated with high uncertainty in the estimation by BSM. The different roles of parameters in eq. (1) is a possible reason why sensitivity and uncertainty do not necessarily correspond to each other. The retardation times $\tau_{\mathrm{S}}$ and $\tau_{\mathrm{V}}$ represent the time constants of the strain recovery curve and are responsible for determining the curvature. Conversely, the stress tensor and pore pressure are coefficients of the curve and are responsible for determining the final amount of recovery.

\section{Conclusion}

In this study, we propose a new ASR analytic procedure that enables us to quantify the uncertainty of the measurement results and examine it using simulated data. We demonstrate that BSM can be used to quantitatively evaluate measurement error as the standard deviation of MPD. The results obtained by the new method reveal that the uncertainty of the estimation results varies with each parameter. In particular, the uncertainty of the non-diagonal elements of the stress tensor $\left(\sigma_{12}, \sigma_{13}\right.$, and $\left.\sigma_{23}\right)$ is smaller than that of the other parameters in the estimation (Case-2) with the known elastic moduli. Sensitivity analysis revealed that this difference is partially impacted by parameter sensitivity. The results suggest that there is not necessarily a correspondence between the results of BSM estimates and sensitivity analysis. Further studies on the relationship between sensitivity and estimation uncertainty are required. Evaluating the applicability of the proposed analytic procedure to real data is the next step in validating this method, which will be the subject of our future research.

\section{Acknowledgement}

This work was partly supported by JSPS Grants-in-Aid for Scientific Research 19H00717 and 20K15219. We would like to express our gratitude for this support.

\section{REFERENCES}

1) M.D. Zoback: Reservoir Geomechanics, (Cambridge University Press, Cambridge, 2007) p. 502.

2) R.S. Stein: Nature 402 (1999) 605-609.

3) B. Amadei and O. Stephansson: Rock stress and Its Measurement, (Springer Science \& Business Media, Berlin, 1997) p. 490.

4) C. Scheidt, L. Li and J. Caers: Quantifying Uncertainty in Subsurface Systems, (John Wiley \& Sons, Hoboken, 2018) p. 304.

5) S. Atkins, A.P. Valentine, P.J. Tackley and J. Trampert: Phys. Earth Planet. Inter. 257 (2016) 171-186.

6) L.F. Contreras, E.T. Brown and M. Ruest: J. Rock Mech. Geotech. Eng. 10 (2018) 11-31.

7) X. Feng and R. Jimenez: Eng. Geol. 199 (2015) 19-27.

8) A.E. Aladejare and Y. Wang: Geotech. Geol. Eng. 37 (2019) 50455060 .

9) K. Matsuki and K. Takeuchi: Int. J. Rock Mech. Min. Sci. Geomech. Abstr. 30 (1993) 1019-1022.

10) W. Lin, E.C. Yeh, H. Ito, T. Hirono, W. Soh, C.Y. Wang, K.F. Ma, J.H. Hung and S.R. Song: Terr. Atmospheric Ocean. Sci. 18 (2007) 379_ 393. 
11) T.B. Byrne, W. Lin, A. Tsutsumi, Y. Yamamoto, J.C. Lewis, K. Kanagawa, Y. Kitamura, A. Yamaguchi and G. Kimura: Geophys. Res. Lett. 36 (2009) L23310.

12) Y. Yamamoto, W. Lin, H. Oda, T. Byrne and Y. Yamamoto: Tectonophysics 600 (2013) 91-98.

13) Y. Nagano, W. Lin and K. Yamamoto: Mar. Pet. Geol. 66 (2015) 418 424.

14) D. Sun, H. Sone, W. Lin, J. Cui, B. He, H. Lv and Z. Cao: Sci. Rep. 7 (2017) 4503.

15) K. Matsuki: Int. J. Rock Mech. Min. Sci. 45 (2008) 952-965.

16) S. Watanabe: Mathematical Theory of Bayesian Statistics, (Chapman and Hall/CRC, London, 2018) p. 320.

17) S. Watanabe: Theory and Method of Bayes Statistics, (Corona Publishing, Tokyo, 2012) pp. 192-202.

18) M.D. Hoffman and A. Gelman: J. Mach. Learn. Res. 15 (2014) 15931623.

19) B.D. Ripley: Stochastic Simulation, (John Wiley \& Sons, Hoboken, 1987) p. 238

20) C.M. Bishop: Pattern Recognition and Machine Learning, (SpringerVerlag, Berlin, 2006) p. 738.
21) A. Gelman and D.B. Rubin: Stat. Sci. 7 (1992) 457-472.

22) A. Gelman, J.B. Carlin, H.S. Stern, D.B. Dunson, A. Vehtari and D.B. Rubin: Bayesian Data Analysis, (Chapman and Hall/CRC, London, 2013) p. 675.

23 ) B. Carpenter, A. Gelman, D.M. Hoffman, D. Lee, B. Goodrich, M. Betancourt, M. Brubaker, J. Guo, P. Li and A. Riddell: J. Stat. Softw. 76 (2017).

24) W. Flügge: Viscoelasticity, (Springer-Verlag, Berlin, 1975) p. 203.

25) N. Kumagai, H. Ito and S. Sasajima: J. Soc. Mater. Sci. Jpn. 35 (1986) 484-489.

26) Y. Kobayashi and H. Ito: J. Soc. Mater. Sci. Jpn. 31 (1982) 790-798.

27) A. Saltelli, M. Ratto, T. Andres, F. Campolongo, J. Cariboni, D. Gatelli, M. Saisana and S. Tarantola: Global Sensitivity Analysis: The Primer, (John Wiley \& Sons, Hoboken, 2008) p. 292.

28) N. Farchmin, M. Hammerschmidt, P.-I. Schneider, M. Wurm, B. Bodermann, M. Bär and S. Heidenreich: Proc. SPIE 11057, Modeling Aspects in Optical Metrology VII (2019).

29) I.M. Sobol': Math. Models Comput. Simul. 2 (1990) 112-118.

30) T. Homma and A. Saltelli: Reliab. Eng. Syst. Saf. 52 (1996) 1-17.

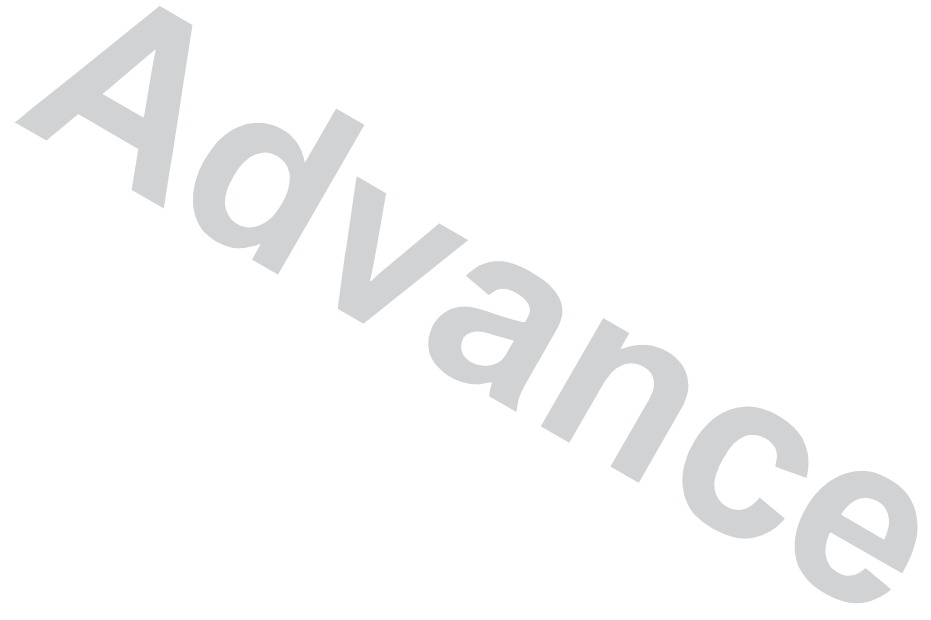

